\title{
STRUCTURE AND THERMAL STABILITY OF TIN BRONZE NANOSTRUCTURED BY HIGH PRESSURE TORSION
}

\author{
A. V. Stolbovsky", V. V. Popov, E. N. Popova \\ M.N. Miheev Institute of Metal Physics of Ural Branch of Russian Academy of Sciences, 18 S. Kovalevskoy st., \\ Ekaterinburg, Russian Federation \\ *Corresponding author. E-mail: stolbovsky@imp.uran.ru; address for correspondence: 18, ul. S. Kovalevskoy, 620990, \\ Ekaterinburg, Russian Federation. Tel.: +7 (343) 378-35-74; fax: (343) 374-52-44
}

The evolution of the structure of bronze containing $7.4 \mathrm{wt}$ \% Sn under severe plastic deformation by high pressure torsion has been studied by transmission electron microscopy and microhardness measurements. The thermal stability of the structures obtained has been investigated in subsequent annealing. It is demonstrated that the presence of an enhanced amount of impurities considerably retards the relaxation processes. It is shown that, along with an enhanced thermal stability of the nanostructures obtained, the transition from the overall growth of crystallite sizes at heating to the dramatic development of recrystallization processes in the alloy under study is much smoother than in pure metals.

Keywords: nanostructuring, nanostructures, severe plastic deformation, high-pressure torsion, grain boundaries, thermal stability, tin bronze.

DOI: $10.17804 / 2410-9908.2015 .5 .118-132$

\section{References}

1. Valiev R.Z. Nanostructuring of metals by severe plastic deformation for advanced properties. Nature Materials, 2004, vol. 3, pp. 511-516. DOI: 10.1038/nmat1180.

2. Valiev R.Z., Estrin Y., Horita Z., Langdon T.G., Zehetbauer M. J., Zhu Y. T. Producing bulk ultrafine-grained materials by severe plastic deformation. The Journal of the Minerals, Metals \& Materials Society, 2006, vol. 58, iss. 4, pp. 33-39. DOI: 10.1007/s11837-006-0213-7.

3. Valiev R.Z., Aleksandrov I.V. Obyomnye nanostrukturnye metallicheskie materialy [Bulk Nanostructured Metal Materials]. M., Akademkniga Publ., 2007, 398 p. ISBN 978-5-94628-217-8. (In Russian).

4. Estrin Y., Vinogradov A. Extreme grain refinement by severe plastic deformation: A wealth of challenging science. Acta Materialia, 2013, vol. 61, iss. 3, pp. 782-817. DOI:10.1016/j.actamat.2012.10.038.

5. Pippan R., Scheriau S., Hohenwarte A., Hafok M. Advantages and limitations of HPT: A review. Materials Science Forum, 2008, vol. 584-586, pp. 16-21. DOI: 10.4028/www.scientific.net/MSF.584-586.16.

6. Vorhauer A., Pippan R. Microstructure and thermal stability of tungsten based materials processed by means of Severe Plastic Deformation Materials Science Forum, 2003, vol. 426-432, pp. 2747-2752. DOI:10.4028/www.scientific.net/MSF.426-432.2747.

7. Sabirov I., Pippan R. Characterization of tungsten fragmentation in a W-25\% Cu composite after high-pressure torsion. Materials Characterization, 2007, vol. 58, iss. 10, pp. 848-853. DOI: 10.1016/j.matchar.2006.08.001.

8. Popov V.V., Valiev R.Z., Popova E.N., Sergeev A.V., Stolbovsky A.V., Kazihanov V.U. Structure and properties of grain boundaries in submicrocrystalline W obtained by severe plastic deformation. Defect and Diffusion Forum, 2009, vol. 283-286, p. 629-638. DOI: 10.4028/www.scientific.net/DDF.283-286.629.

9. Popov V.V., Grabovetskaya G.P., Sergeev A.V., Mishin I.P., Timofeev A.N., Kovalenko E.V. Structure and properties of grain boundaries in submicrocrystalline molybdenum 
prepared by high-pressure torsion. The Physics of Metals and Metallography, 2010, vol. 109, iss. 5, pp. 556-562. DOI: 10.1134/S0031918X10050169.

10. Popova E.N., Popov V.V., Romanov E.P., Pilyugin V.P. Thermal stability of nanocrystalline $\mathrm{Nb}$ produced by severe plastic deformation. The Physics of Metals and Metallography, 2006, vol. 101, iss. 1, pp. 52-67. DOI: 10.1134/S0031918X06010078.

11. Popova E.N., Popov V.V., Romanov E.P., Pilyugin V.P. Effect of the degree of deformation on the structure and thermal stability of nanocrystalline niobium produced by high-pressure torsion.

The Physics of Metals and Metallography, 2007, vol. 103, iss. 4, pp 407-413. DOI: 10.1134/S0031918X0704014X.

12. Popov V.V., Popova E.N., Stolbovskiy A.V. Nanostructuring Nb by various techniques of severe plastic deformation. Materials Science and Engineering: A, 2012, vol. 539, pp. 22-29. DOI:10.1016/j.msea.2011.12.082.

13. Schafler E., Pippan R. Effect of thermal treatment on microstructure in high pressure torsion (HPT) deformed nickel. Materials Science and Engineering: A, 2004, vol. 387-389, pp. 799-804. DOI:10.1016/j.msea.2004.01.112.

14. Zhang H.W., Huang X., Pippan R., Hansen N. Thermal behavior of Ni $(99.967 \%$ and $99.5 \%$ purity) deformed to an ultra-high strain by high pressure torsion. Acta Materialia, 2010, vol. 58, iss. 5, pp. 1698-1707. DOI:10.1016/j.actamat.2009.11.012.

15. Pilyugin V.P., Gapontseva T.M., Chashchukhina T.I., Voronova L.M., Shchinova L.I., Degtyarev M.V. Evolution of the structure and hardness of nickel upon cold and low-temperature deformation under pressure. The Physics of Metals and Metallography, 2008, vol. 105, iss. 4, pp. 409-419. DOI: 10.1134/S0031918X08040157.

16. Popov V.V., Popova E.N., Stolbovskii A.V., Pilyugin V.P., Arkhipova N.K. Nanostructurization of $\mathrm{Nb}$ by high-pressure torsion in liquid nitrogen and the thermal stability of the structure obtained. The Physics of Metals and Metallography, 2012, vol. 113, iss. 3, pp. 295-301. DOI: $10.1134 / \mathrm{S} 0031918 X 1203009 X$.

17. Popov V.V., Popova E.N., Stolbovskiy A.V., Pilyugin V.P. Thermal stability of nanocrystalline structure in niobium processed by high pressure torsion at cryogenic temperatures. Materials Science and Engineering: A, vol. 528, iss. 3, pp. 1491-1496. DOI:10.1016/j.msea.2010.10.052.

18. Rathmayr G.B., Pippan R. Influence of impurities and deformation temperature on the saturation microstructure and ductility of HPT-deformed nickel. Acta Materialia, 2011, vol. 59, iss. 19, pp. 7228-7240. DOI: 10.1016/j.actamat.2011.08.023.

19. Popov V.V., Popova E.N., Kuznetsov D.D., Stolbovskii A.V., Pilyugin V.P. Thermal stability of nickel structure obtained by high-pressure torsion in liquid nitrogen. The Physics of Metals and Metallography, 2014, vol. 115, iss. 7, pp. 682-691. DOI: 10.1134/S0031918X14070060.

20. Popov V.V., Stolbovsky A.V., Popova E.N., Pilyugin V.P. Structure and thermal stability of $\mathrm{Cu}$ after severe plastic deformation. Defect and Diffusion Forum, 2010, vol. 297-301, pp. 1312-1321. DOI:10.4028/www.scientific.net/DDF.

21. Stolbovsky A.V., Popov V.V., Popova E. N., Pilyugin V.P. Structure, thermal stability, and state of grain boundaries of copper subjected to high-pressure torsion at cryogenic temperatures. Bulletin of the Russian Academy of Sciences: Physics, 2014, vol. 78, iss. 9, pp. 908-916. DOI: $10.3103 / \mathrm{S} 1062873814090299$.

22. Oh-ishi K., Horita Z., Smitz D.J., Valiev R.Z., Nemoto M., Langdon. T.G. Fabrication and thermal stability of a nanocrystalline $\mathrm{Ni}-\mathrm{Al}-\mathrm{Cr}$ alloy: comparison with pure $\mathrm{Cu}$ and $\mathrm{Ni}$. Journal of Materials Research, 1999, vol. 14, iss. 11, pp. 4200-4207.

23. Pippan R., Scheriau S., Taylor A., Hafok M., Hohenwarter A., Bachmaier A. Saturation of fragmentation during severe plastic deformation. Annual Review of Materials Research, 2010, vol. 40, pp. 319-343. DOI: 10.1146/annurev-matsci-070909-104445.

24. Konkova T.N., Mironov S.Y., Korznikov A.V. Abnormal grain growth in cryogenically deformed copper. Fizicheskaya mezomekhanika, 2011, vol. 14, no. 4, pp. 29-38. (In Rusian). 
open-access journal

25. Konkova T.N., Mironov S.Y., Korznikov A.V. Room-temperature instability of the structure of copper deformed at a cryogenic temperature. Russian metallurgy (Metally), 2011, vol. 2011, iss. 7, pp. 689-698. DOI: 10.1134/S0036029511070081.

26. Popova E.N., Romanov E.P., Sudareva S.V. A15 superconducting composites and highstrength $\mathrm{Cu}-\mathrm{Nb}$ conductors. The Physics of Metals and Metallography, 2003, vol. 96, iss. 2, pp. 146-159.

27. Popova E.N., Rodionova L.A., Sudareva S.V., Romanov E.P., Khlebova N.E., Chukin A.M. Influence of Different Deformation Techniques on the Structure of Bronze Matrix in Multifilamentary $\mathrm{Nb}_{3} \mathrm{Sn}$ Composites. The Physics of Metals and Metallography, 1993, vol. 76, iss. 2, pp. 228-234.

28. Zhilyaev A.P., Lee S., Nurislamova G.V., Valiev R.Z., Langton T.G.. Microhardness and microstructural evolution in pure nickel during high-pressure torsion. Scripta Materialia, 2001, vol. 44, iss. 12, pp. 2753-2758. DOI:10.1016/S1359-6462(01)00955-1.

29. Tyumentsev, A.N., Ditenberg, I.A., Pinzhin, Yu.P., Korotaev, A.D., and Valiev, R.Z. Microstructure and Mechanisms of its Formation in Submicrocrystalline Copper Produced by Severe Plastic Deformation. The Physics of Metals and Metallography, 2003, vol. 96, iss. 4, pp. 378-387.

30. Hebesberger T., Stuwe H.P., Vorhauer A., Wetscher F., Pippan R.. Structure of Cu deformed by high pressure torsion. Acta Materialia, 2005, vol. 53, iss. 2, pp. 393-402. DOI: $10.1016 /$ j.actamat.2004.09.043. 
Подана в журнал: 17.09 .2015

УДК 669-179:539.374:620.186:620.187.3:669.35'6

DOI: $10.17804 / 2410-9908.2015 .5 .118-132$

\title{
СТРУКТУРА И ТЕРМИЧЕСКАЯ СТАБИЛЬНОСТЬ ОЛОВЯНИСТОЙ БРОНЗЫ, НАНОСТРУКТУРИРОВАННОЙ МЕТОДОМ КРУЧЕНИЯ ПОД ВЫСОКИМ ДАВЛЕНИЕМ
}

\author{
А. В. Столбовский ${ }^{*}$ В. В. Попов, Е. Н. Попова \\ Федеральное государственное бюджетное учреждение науки Институт физики металлов имени \\ М.Н. Михеева Уральского отделения Российской академии наук, ул. С. Ковалевской, 18, Екатеринбург, \\ Российская Федерация \\ *Ответственный автор. Электронная почта: stolbovsky@imp.uran.ru; адрес для переписки: 620990, \\ ул. С. Ковалевской, 18, Екатеринбург, Российская Федерация. \\ Телефон: +7 (343) 378-35-74; факс: (343) 374-52-44
}

Методами просвечивающей электронной микроскопии и измерения микротвердости исследована эволюция структуры бронзы с содержанием 7,4 масс. \% олова при интенсивной пластической деформации кручением под высоким давлением при комнатной температуре. Изучена термическая стабильность получаемой структуры при последующих отжигах. Показано, что повышенное содержание примесей существенно замедляет релаксационные процессы. Установлено, что наряду с повышенной термической стабильностью полученной наноструктуры, переход от равномерного общего увеличения размеров кристаллитов при нагреве к резкому развитию рекристаллизационных процессов в изучаемом сплаве заметно более плавный, чем в чистых металлах.

Ключевые слова: наноструктурирование, наноструктуры, интенсивная пластическая деформачия, кручение под высоким давлением, гранищы зерен, термическая стабильность, оловянистая бронза.

\section{1. Введение}

В настоящее время наноструктурные и субмикрокристаллические материалы привлекают большое внимание исследователей благодаря своему необычному механическому поведению и уникальным структуре и свойствам. Многочисленными исследованиями установлено, что объемные металлические наноструктурные материалы можно получать различными способами интенсивной пластической деформации (ИПД) [1-4]. Одним из преимуществ материалов, наноструктурированных интенсивной пластической деформацией, по сравнению с нанокристаллическими материалами, получаемыми с использованием порошковых технологий, является полное отсутствие пористости. Среди наиболее популярных способов ИПД особое место занимает кручение под высоким давлением (КВД) [5]. Этот метод позволяет проводить непрерывную деформацию материалов, даже хрупких и прочных, до очень высоких степеней, причем возможно изменять температуру деформирования в широком диапазоне. Для более твердых и хрупких металлов, например таких, как вольфрам и молибден, КВД проводят при повышенных температурах, что позволяет получать субмикрокристаллическую структуру [6-9]. КВД при комнатной температуре позволяет получать близкую к нанокристаллической структуру в $\mathrm{Nb}$ [10-12] и субмикрокристаллическую структуру в $\mathrm{Ni}$ [13-15], а проведение КВД при криогенной температуре позволило получить истинную нанокристаллическую структуру в ряде чистых металлов $(\mathrm{Nb}, \mathrm{Ni}$ и технически чистая медь) [16-21]. 
Одной из важнейших проблем в создании наноструктурированных материалов с особыми свойствами является низкая термическая стабильность получаемых структур, особенно в чистых металлах [22, 23]. Как показано в работе [20], в высокочистой меди КВД при комнатной температуре позволяет получить крайне неоднородную субмикрокристаллическую структуру, измельчению которой препятствуют процессы динамического возврата и рекристаллизации. При криогенной температуре структура получается более однородной и дисперсной, но она крайне нестабильна, и кристаллиты растут даже при отогреве до комнатной температуры, т.е. происходит постдинамическая рекристаллизация. В меди технической чистоты присутствие примесей замедляет релаксационные процессы и, как следствие, способствует измельчению структуры, но даже в этом случае постдинамическая рекристаллизация не позволяет сохранить наноструктуру, полученную КВД при температуре жидкого азота $[20,21]$. В связи с этим некоторые авторы вообще ставят под сомнение вопрос о целесообразности использования ИПД для формирования нанокристаллической структуры в меди $[24,25]$.

Представлялось интересным выяснить, возможно ли получить нанокристаллическую структуру и существенно повысить ее термическую стабильность за счет значительно более высокого содержания примесей, в частности, за счет легирования меди оловом в количестве нескольких процентов. Поэтому в настоящей работе изучено воздействие КВД и последующих отжигов на оловянистую бронзу $\mathrm{Cu}-7,4$ \% $\mathrm{Sn}$, представляющую твердый раствор олова в меди.

\section{2. Материал и методика исследования}

В настоящей работе для исследования была выбрана бронза с содержанием 7,4 масс. \% олова. Этот сплав представляет интерес не только как твердый раствор на основе меди, на котором будет сделана попытка преодолеть проблемы, возникающие при наноструктурировании этого металла. Оловянистая бронза является матрицей при создании сверхпроводящих композитов на основе соединения $\mathrm{Nb}_{3} \mathrm{Sn}$ [26], в связи с чем ее изучение при различных экстремальных воздействиях является исключительно важным. Сплав $\mathrm{Cu}-7,4 \% \mathrm{Sn}$ в литом состоянии подвергли гомогенизирующему отжигу в вакуумной печи при температуре $750{ }^{\circ} \mathrm{C}$ в течение 2 ч, чтобы исключить влияние дендритной ликвации и выровнять состав по сечению образцов. Для деформации кручением под высоким давлением вырезали диски диаметром 10 мм и толщиной 0,5 мм. Полученные диски деформировали в наковальнях Бриджмена под давлением 4 ГПа с угловой скоростью 0,3 об/мин на 0,25; 0,5; 1 и 5 оборотов при комнатной температуре. Истинную деформацию (e) рассчитывали как сумму истинной деформации сдвигом $e_{c д s}=\ln \sqrt{1+\left(\frac{\varphi \cdot R}{h_{k}}\right)^{2}}$ и осадкой $e_{\text {осад }}=\ln \frac{h_{0}}{h_{K}}$, где $\varphi-$ угол скручивания (рад), $R$ - расстояние от оси вращения (мм), а $h_{0}$ и $h_{k}$ - толщина образца до и после деформации [10]. Поскольку при КВД деформация распределена неравномерно, увеличиваясь от центра образцов к краю, далее анализировали и сравнивали структуру и микротвердость на середине радиуса дисков.

Для изучения термической стабильности полученной структуры некоторые образцы отжигали при температурах от 200 до $400{ }^{\circ} \mathrm{C}$ с выдержкой 1 ч. Все отжиги проводили в вакуумной трубчатой печи при давлении $10^{-2}-10^{-3}$ Па.

Структуру деформированных и отожженных образцов изучали на просвет в электронных микроскопах Philips-CM30 SuperTwin и JEM-200CX с дальнейшей обработкой снимков в автоматизированном комплексе SIAMS-600 для построения распределения зерен по размерам. Микротвердость измеряли в оптическом комплексе NEOPHOT-21 с четырёхгранной пирамидкой Викерса и рассчитывали по формуле $H=\frac{18192 \cdot P}{D^{2}}$, МПа, где $P-$ 
нагрузка в граммах, а $D$ - диагональ отпечатка в микронах. Применяемая нагрузка составляла 70 г. Каждое значение $D$ рассчитано как среднее по всем отпечаткам (не менее 9). Погрешность измерения микротвердости составила в среднем 2-3\% и не была выше $5 \%$ при доверительной вероятности 0,95 .

\section{3. Результаты исследования и их обсуждение}

Поскольку, как отмечено выше, оловянистые бронзы широко используются при создании многоволоконных сверхпроводящих композитов на основе соединения $\mathrm{Nb}_{3} \mathrm{Sn}$, при производстве которых применяют большую пластическую деформацию многократным холодным волочением с промежуточными отжигами, то механизмы их деформации в условиях композита (в присутствии ниобиевых волокон) достаточно хорошо изучены [27]. Представлялось интересным выяснить поведение этого сплава при ИПД кручением под высоким давлением, выявить возможность его наноструктурирования и исследовать термическую стабильность получаемых структур.

На рис. 1 показаны зависимости микротвердости бронзы от расстояния степени деформации при комнатной температуре. Многие экспериментальные данные указывают на неравномерное распределение деформации по радиусу образца при КВД [28-30], хотя с увеличением степени деформации кручением под давлением (с ростом числа оборотов наковальни) эта неоднородность структуры и свойств (в частности микротвердости) выравнивается. Эта закономерность сохраняется и в рассматриваемом случае, как видно из рис. 1, где представлено изменение микротвердости по радиусу образцов бронзы после КВД с разными степенями деформации (расчётные значения на середине радиуса, $x=2,5$, составляли от $e=2,9$ для 0,25 об. до $e=7,8$ для 5 об.). Деформирование проводилось при комнатной температуре. При небольших деформациях (малых углах поворота наковален) разница в микротвердости в центре и на периферии так же значительна, как и, например, в случае чистого ниобия [11], однако выравнивание микротвердости по всему сечению образцов происходит при гораздо меньших степенях деформации по сравнению с ниобием. Уже при деформации на 1 оборот $(e=5,3)$ достигается насыщение, и дальнейшее увеличение степени деформации не приводит к росту микротвердости. Отметим, что в ниобии насыщение, при котором структура больше не измельчается, а микротвердость не увеличивается с ростом степени деформации, наступает при КВД на 5 оборотов [11].

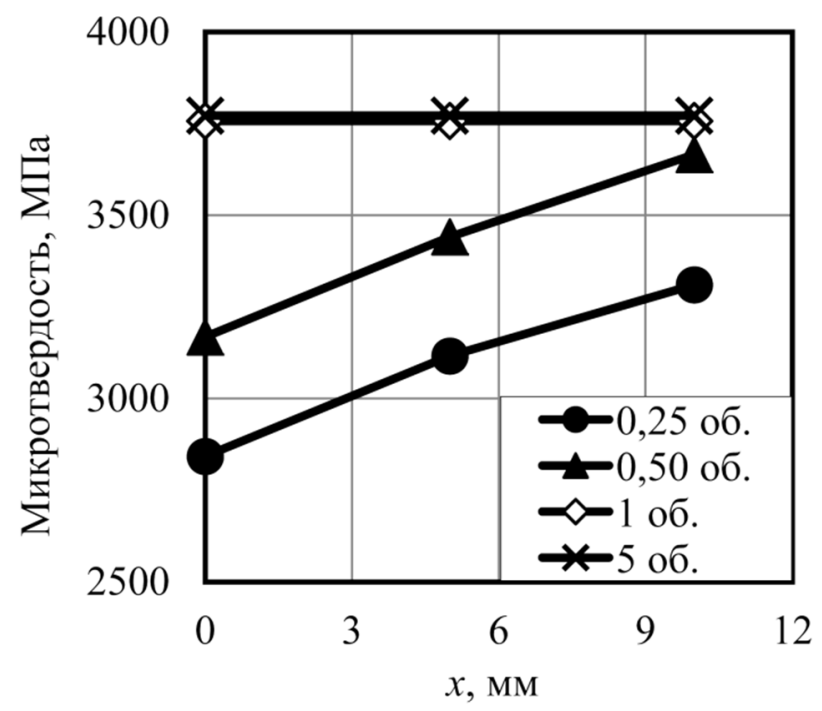

Рис. 1. Изменение микротвердости оловянистой бронзы по радиусу образцов $(x-$ расстояние от центра образца) при разных степенях деформации КВД 
В исследуемой бронзе значение микротвердости на стадии насыщения достигает 3750 МПа, что существенно выше, чем значение для меди технической чистоты в нанокристаллическом состоянии (около $2100 \mathrm{MПа)} \mathrm{[20,} \mathrm{21].}$

На рис. 2 представлены электронные микрофотографии структуры бронзы после 1 и 5 оборотов деформации кручением под высоким давлением при комнатной температуре. Структура бронзы после достижения деформации в 1 оборот уже не отличается от структуры при значительно большем числе оборотов. Это согласуется с данными по микротвердости (см. рис. 1) и можно утверждать, что и измельчение структуры выходит на насыщение уже при деформации на 1 оборот. Полученная структура является нанокристаллической, со средним размером зерен 65-70 нм.

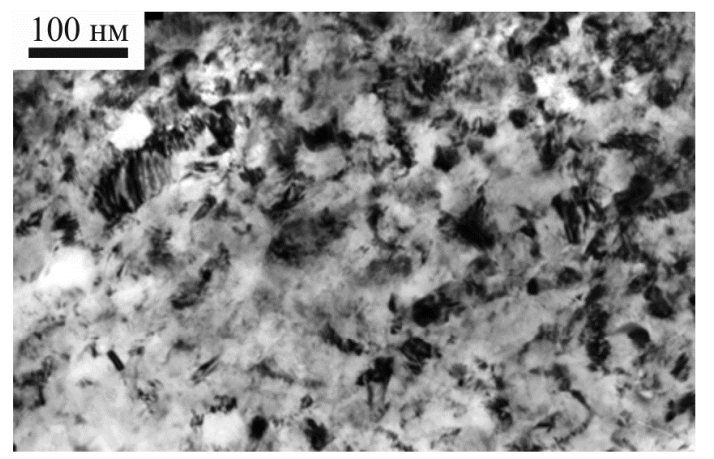

$a$

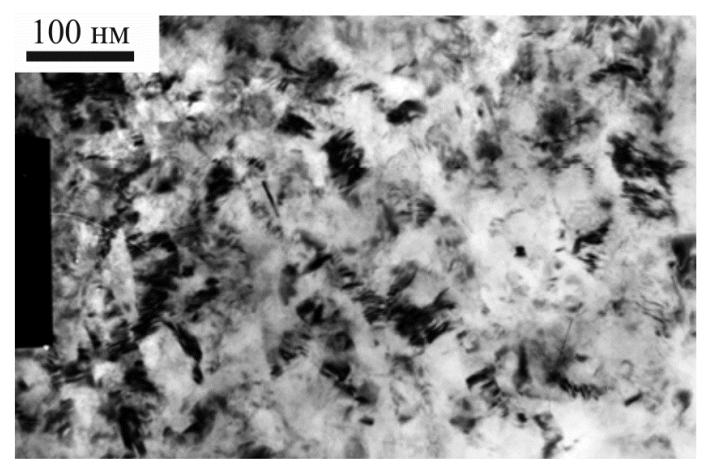

B

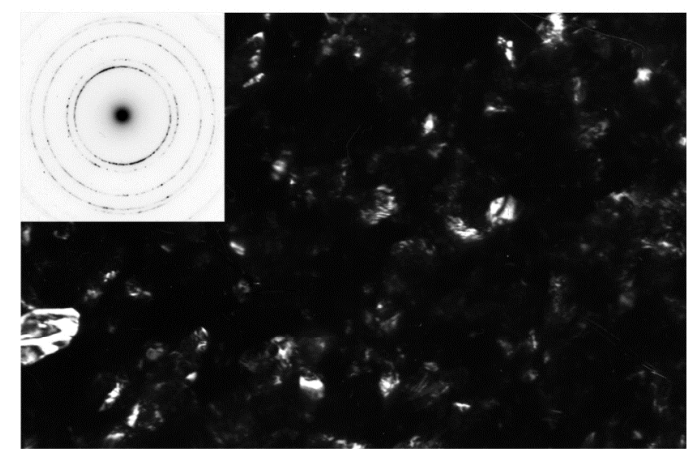

б

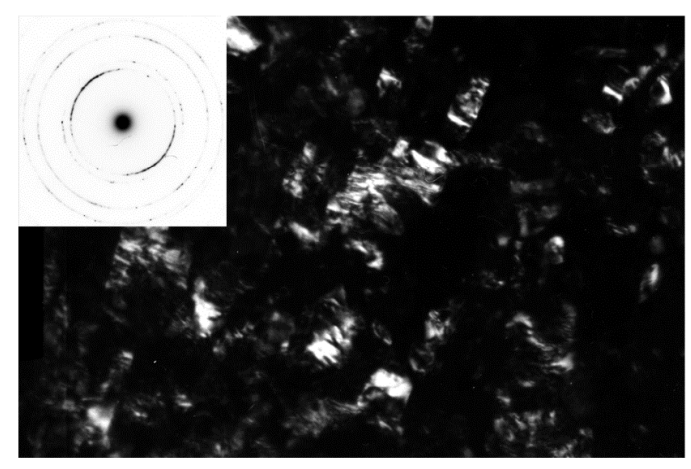

2

Рис. 2. Структура бронзы после КВД на 1 оборот $(a, \sigma)$ и на 5 оборотов $(b, 2)$ :

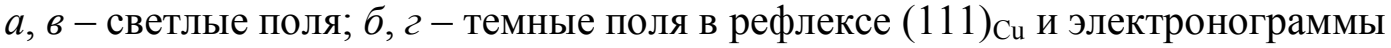

На рис. 3 представлены гистограммы распределения зерен по размерам для 1 и 5 оборотов КВД. Хорошо видно, что эти гистограммы идентичны, они одномодальные, с узким распределением, указывающим не только на однородность структуры, но и на отсутствие признаков рекристаллизации, в отличие от того, что наблюдалось при деформировании меди $[20,21]$. Важно отметить, что данные структуры, в отличие от меди, стабильны при длительном вылеживании при комнатной температуре.

Представлялось интересным изучить термическую стабильность полученных нанокристаллических структур, т. е. их эволюцию при нагреве.

Хотя структурное состояние образцов бронзы, деформированных на 1 и 5 оборотов, практически одинаково, их термическая стабильность может различаться, как это наблюдалось, например, в чистых $\mathrm{Nb}$ и $\mathrm{Cu}$, где было обнаружено, что после более интенсивной деформации структура становится менее стабильной при последующем нагреве $[11,20]$. Поэтому, несмотря на идентичность полученных после разных степеней деформации структур бронзы, были исследованы образцы после разных степеней деформации КВД, чтобы определить влияние степени деформации на их термическую стабильность.

Stolbovsky A. V. et al. / Structure and thermal stability of tin bronze nanostructured by high pressure torsion 


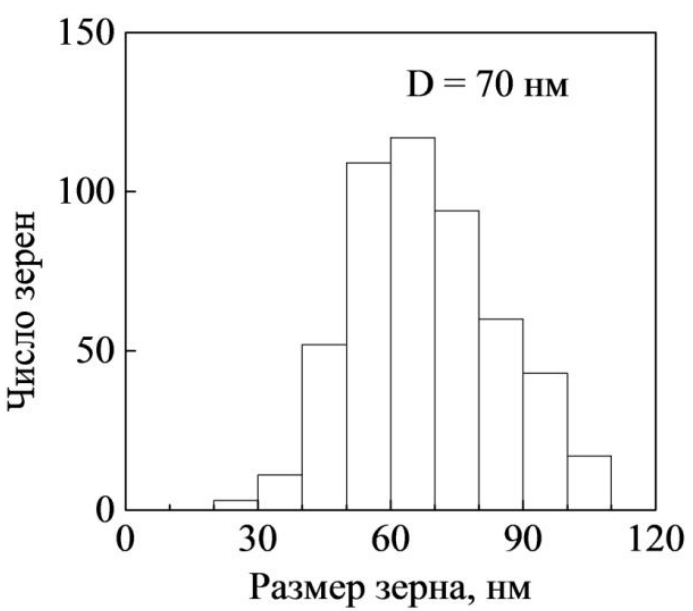

$a$

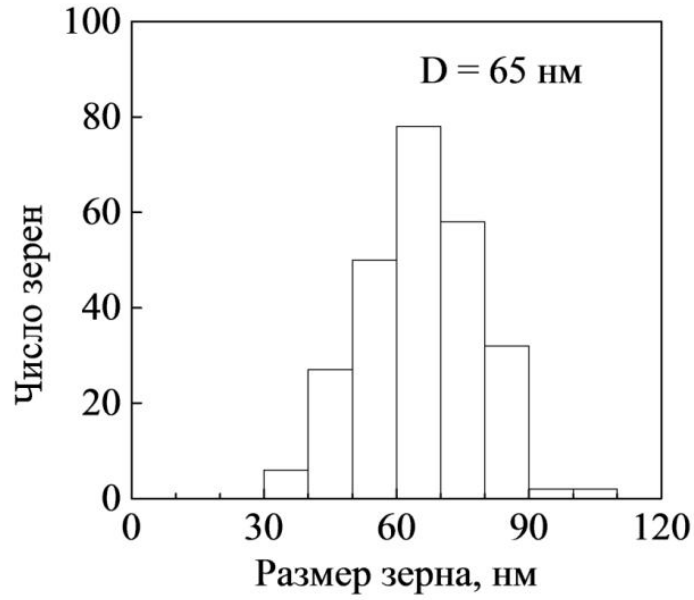

б

Рис. 3. Гистограммы распределения зерен по размерам в бронзе после КВД при комнатной температуре на 1 оборот ( $a$ ) и на 5 оборотов (б)

Обнаружено, что микротвердость образцов оловянистой бронзы, подвергнутых КВД на 1 и 5 оборотов при комнатной температуре, при последующем нагреве изменяется в целом одинаково (рис. 4), т. е. поведение данного сплава несколько отличается от поведения ниобия и меди, в которых с увеличением степени деформации термическая стабильность структуры уменьшалась.

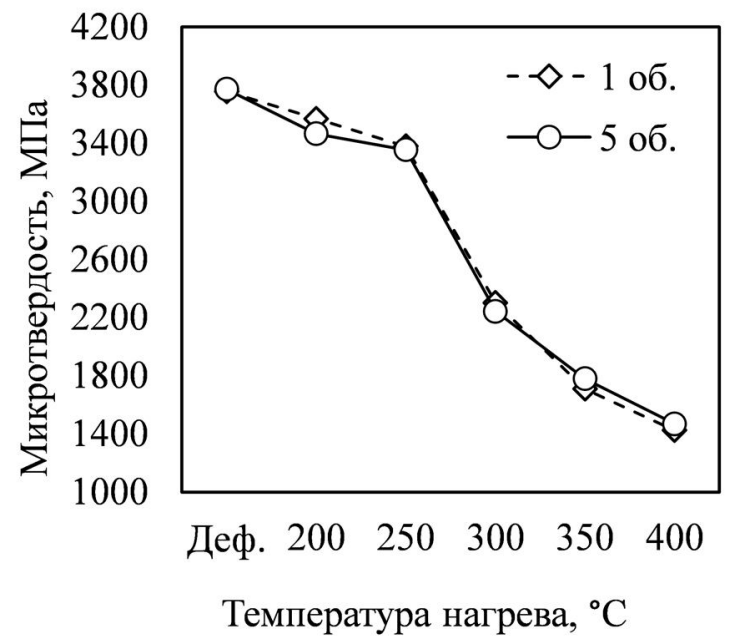

Рис. 4. Микротвердость оловянистой бронзы после КВД на 1 и 5 оборотов и последующего отжига

На рис. 4 хорошо видны два этапа изменения микротвердости. На первом низкотемпературном этапе (при отжигах в интервале $200-250^{\circ} \mathrm{C}$ ) микротвердость снижается плавно и не очень сильно. Для второго этапа, который начинается от температуры $250{ }^{\circ} \mathrm{C}$, характерно резкое падение микротвердости с постепенным выходом на более пологое убывание ее значений при температурах нагрева выше $300^{\circ} \mathrm{C}$.

Исследования структуры показали, что после отжига при температуре $200{ }^{\circ} \mathrm{C}$ структура и размер кристаллитов изменяются незначительно по сравнению с исходным деформированным состоянием. В большинстве зерен виден смазанный искривленный контраст, характерный для деформированного кручением состояния, а все электронограммы - кольцевые, с большим количеством рефлексов на дебаевских кольцах (рис. 5 6, г). Снижение твердости в данном случае, по-видимому, обусловлено протеканием процессов возврата, по- 
opten-aceESS journal

скольку заметных изменений в структуре не происходит, хотя можно отметить несколько более четкие границы кристаллитов.
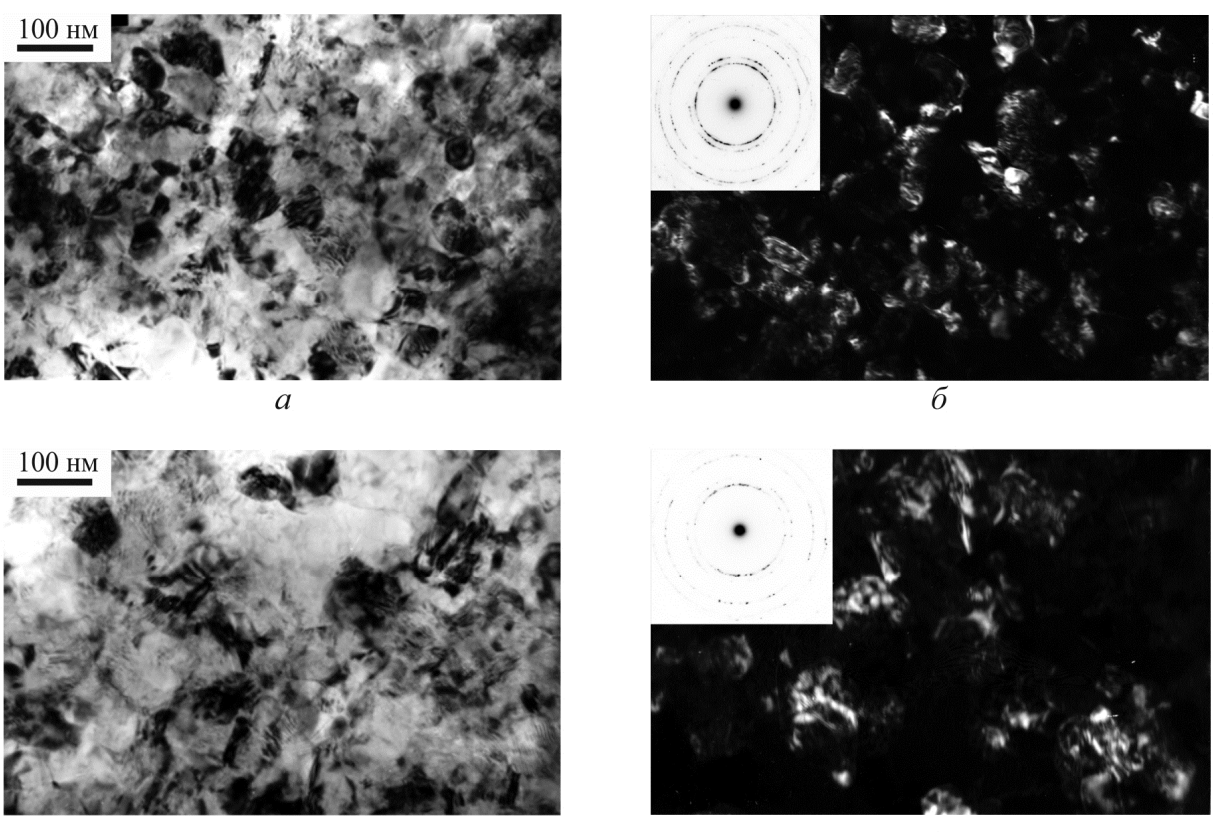

Рис. 5. Структура бронзы после КВД на $1(a, \sigma)$ и $5(b, 2)$ оборотов и отжига при $200{ }^{\circ} \mathrm{C}$ :

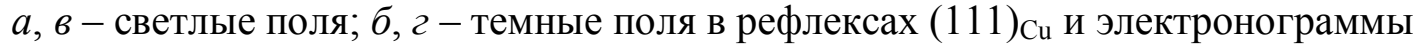

Повышение температуры отжига до $250{ }^{\circ} \mathrm{C}$ также не приводит к существенным изменениям параметров структуры, хотя уже заметно некоторое увеличение среднего размера зерен (рис. 6). Границы зерен несколько выпрямляются и становятся более четкими, хотя характерный для деформированного состояния искривленный контраст в теле некоторых зерен еще достаточно сильно выражен, как и при более низкой температуре отжига, и на электронограммах значительных изменений не наблюдается, т. е. сохраняется большое число рефлексов на Дебаевских кольцах.

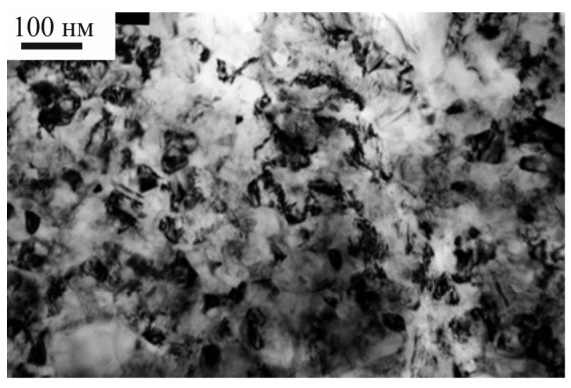

$a$

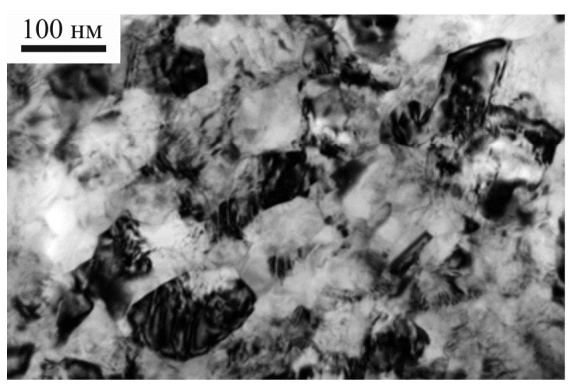

B
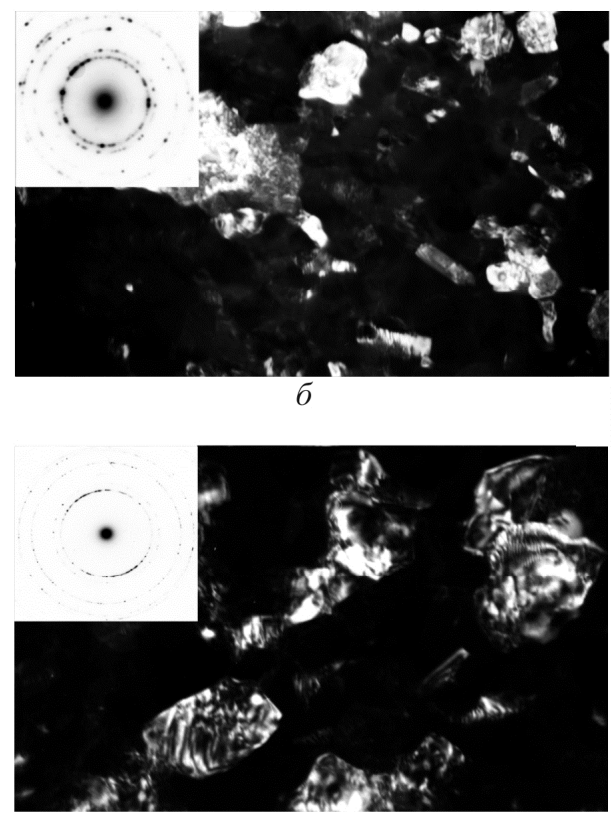

2

Рис. 6. Структура бронзы после КВД на $1(a, \sigma)$ и $5(b, 2)$ оборотов и отжига при $250{ }^{\circ} \mathrm{C}$ : $a$, - - светлые поля; $\sigma, z$ - темные поля в рефлексах (111) Си и электронограммы

Stolbovsky A. V. et al. / Structure and thermal stability of tin bronze nanostructured by high pressure torsion 
Дальнейшее увеличение температуры отжига приводит к резким изменениям в структуре. Так, при температуре отжига $300{ }^{\circ} \mathrm{C}$ наблюдается интенсивный рост зерен и структура становится субмикрокристаллической с размером кристаллитов более 200 нм (рис. 7). При этом формируются двойники отжига, характерные для рекристаллизованной структуры медных сплавов. Границы зерен полностью выпрямляются и наиболее часто наблюдаются стыки зерен под углом в $120^{\circ}$. На электронограммах присутствуют отражения от одной или нескольких плоскостей, что также указывает на полностью рекристаллизованную структуру. Таким образом, можно заключить, что начиная с температуры отжига $300{ }^{\circ} \mathrm{C}$ структура становится полностью рекристаллизованной. Увеличение температуры отжига до $350{ }^{\circ} \mathrm{C}$ приводит к дальнейшему росту размеров кристаллитов и структура приближается к микрокристаллической (рис. 8).
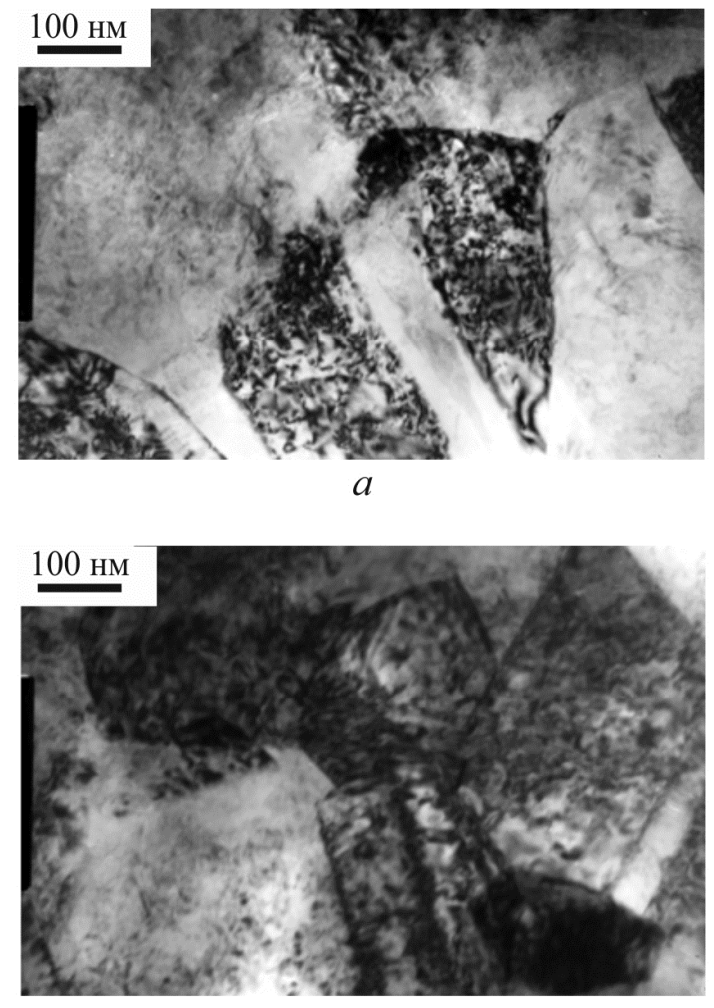

B
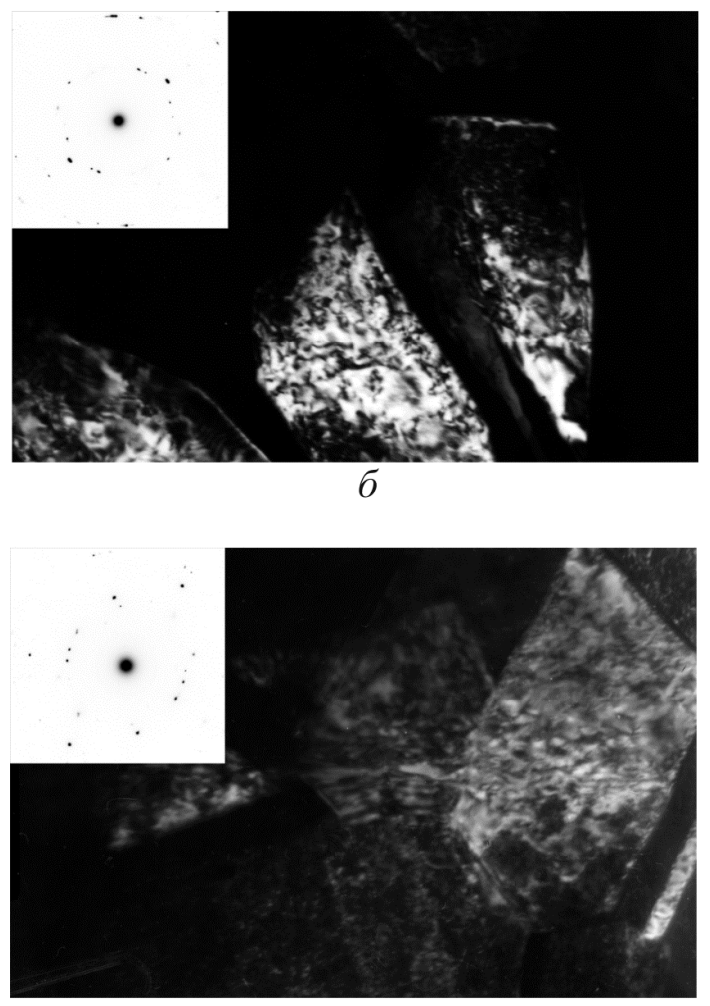

2

Рис. 7. Структура бронзы после КВД на $1(a, \sigma)$ и $5(6,2)$ оборотов и отжига при $300^{\circ} \mathrm{C}$ :

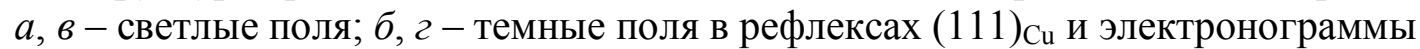

Анализ полученных снимков с построением гистограмм распределения зерен по размерам наглядно демонстрирует изменение структуры при отжиге (рис. 9). Средний размер кристаллитов очень незначительно увеличивается от 65-70 нм в образцах, продеформированных на 1 и 5 оборотов, до 80-90 нм в образцах, отожженных при $200{ }^{\circ} \mathrm{C}$ в течение 1 ч, что свидетельствует об относительно хорошей термической стабильности получаемых структур по сравнению с чистой и технически чистой медью. Процессы, происходящие в деформированной бронзе при нагреве до $200{ }^{\circ} \mathrm{C}$, являются в основном релаксационными.

Нагрев до $250{ }^{\circ} \mathrm{C}$ приводит к увеличению среднего размера кристаллитов до $100 \mathrm{Hм}$ для 1 оборота и 110 нм для 5 оборотов, однако распределение все еще является узким и однородным, без явных признаков рекристаллизации, т.е. без появления комплекса структурных элементов другой (рекристаллизационной) природы, в том числе отдельных зерен аномально больших размеров, появление которых привело бы к бимодальному распределению по размерам. 
В случае нагрева до $300{ }^{\circ} \mathrm{C}$ наблюдается резкое изменение в распределениях зерен по размерам, как для КВД на 1 оборот (рис. 9 в), так и для КВД на 5 оборотов (рис. 9 г) - распределения становятся неоднородными, присутствуют зерна с аномально большими размерами 300-500 нм при среднем размере кристаллитов 230 нм для 1 оборота и 260 нм для 5 оборотов, что также свидетельствует о начале и интенсивном протекании рекристаллизационных процессов.
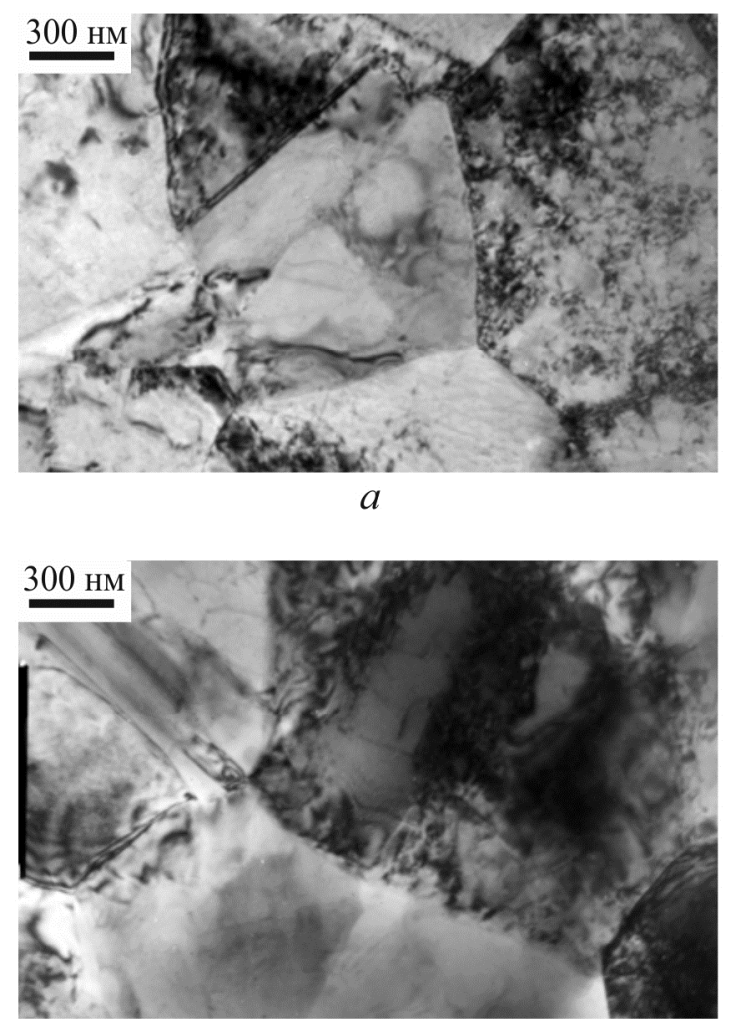

$B$
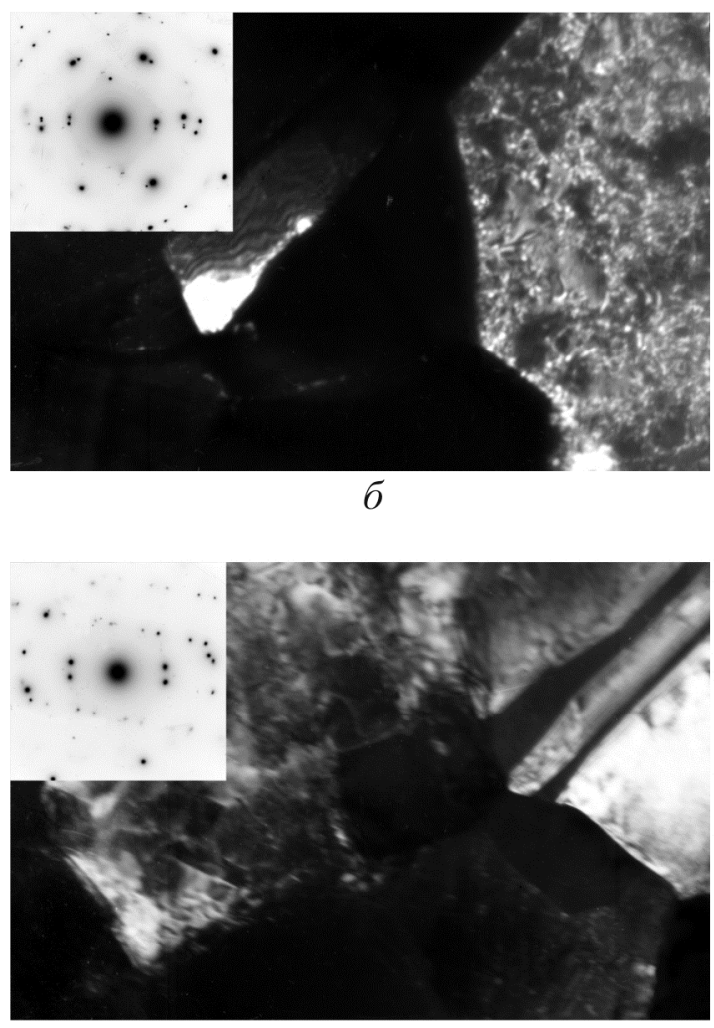

2

Рис. 8. Структура бронзы после КВД на $1(a, \sigma)$ и $5(b, 2)$ оборотов и отжига при $350{ }^{\circ} \mathrm{C}$ :

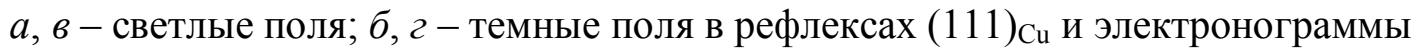

Таким образом, проведенные исследования показывают, что при наноструктурировании оловянистой бронзы методом КВД при комнатной температуре признаки динамической рекристаллизации не наблюдаются и получаемая нанокристаллическая структура стабильна как при комнатной температуре, так и при нагреве до $200{ }^{\circ} \mathrm{C}$.

Нагрев до $250{ }^{\circ} \mathrm{C}$ также не приводит к интенсивному протеканию рекристаллизации, однако наблюдается некоторый рост среднего размера зерен и структура становится субмикрокристаллической. В то же время статистический анализ выявил некоторые различия в средних размерах структурных элементов для 1 и 5 оборотов, хотя на графиках микротвердости различия между образцами, продеформированными на разные степени деформации, не прослеживается. Так, в образце, продеформированном на меньшее количество оборотов, при исходной (после деформации) структуре со средним размером кристаллитов 70 нм против 65 нм для образца с большей деформацией (рис. 3), средний размер кристаллитов при нагреве на каждой температуре отжига несколько меньше, чем в более сильно деформированном образце (рис. 9). Начиная с нагрева на $200{ }^{\circ} \mathrm{C}$ (рис. $9 a$, б) разница в среднем размере кристаллитов составляет 10 нм и остается такой же при отжиге $250{ }^{\circ} \mathrm{C}$ (рис. 9 в, г), а при нагреве на $300{ }^{\circ} \mathrm{C}$ она увеличивается до 30 нм (рис. $9 \partial, e$ ). Это указывает на несколько меньшую терми- 
ческую стабильность образцов с большей деформацией, как и в случае ниобия [11] и меди $[20,21]$, хотя для бронзы это различие не столь сильно выражено.
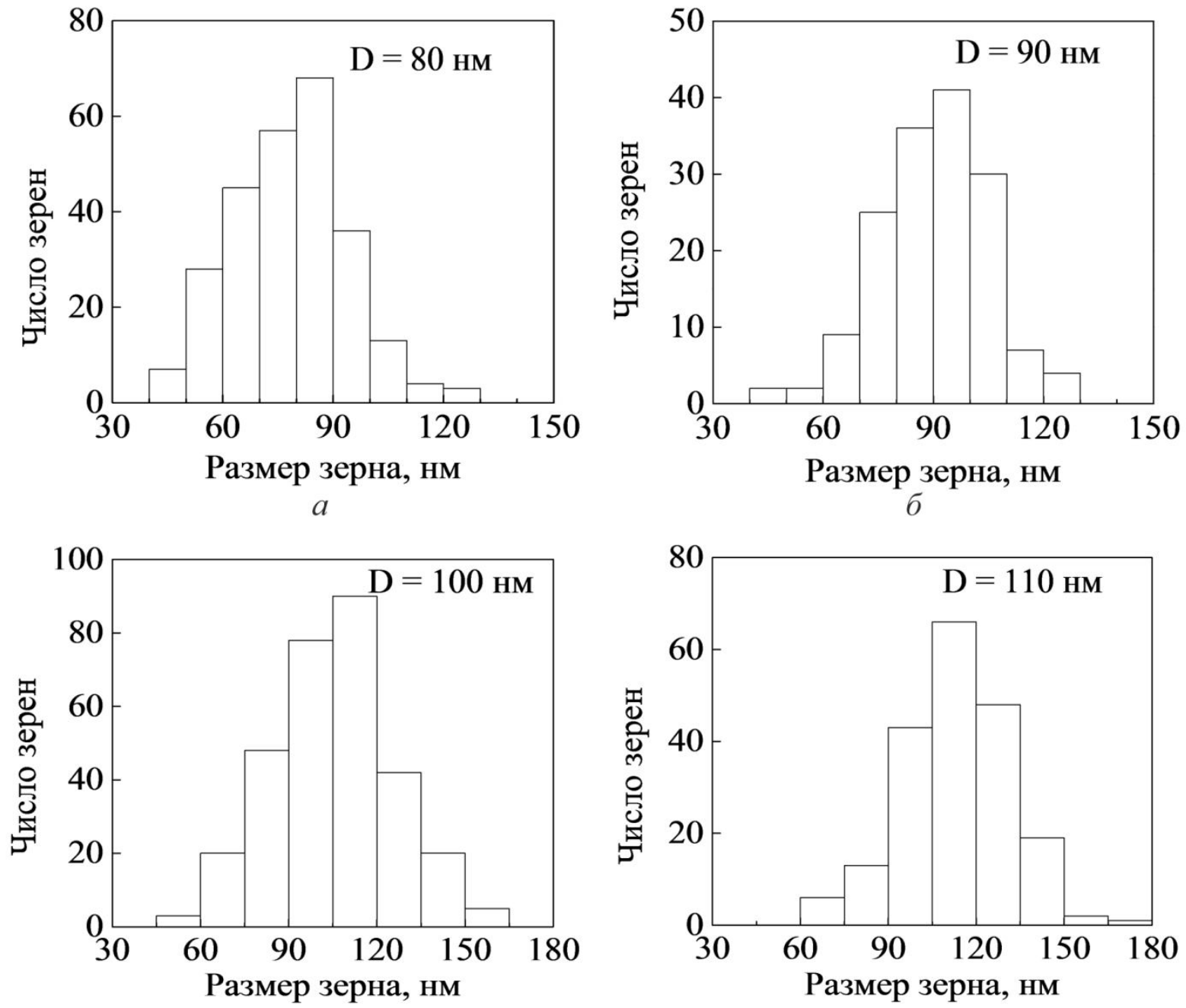

B

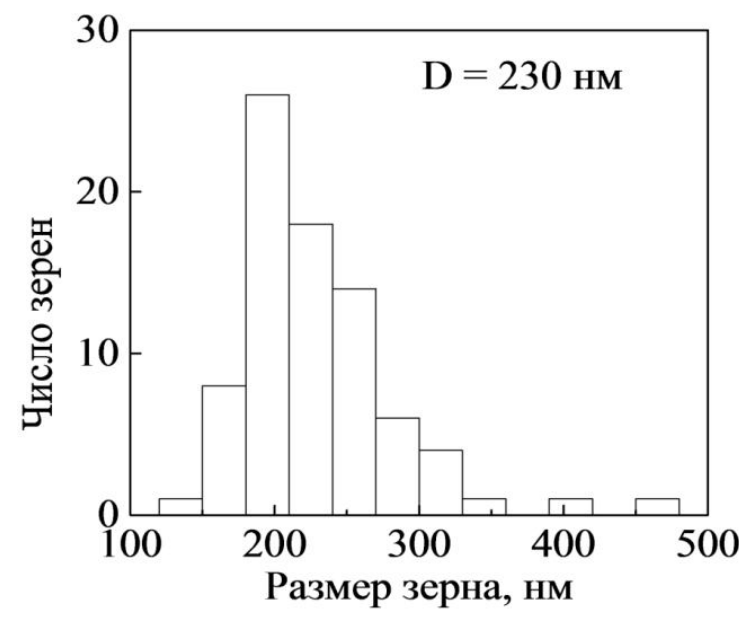

$\partial$

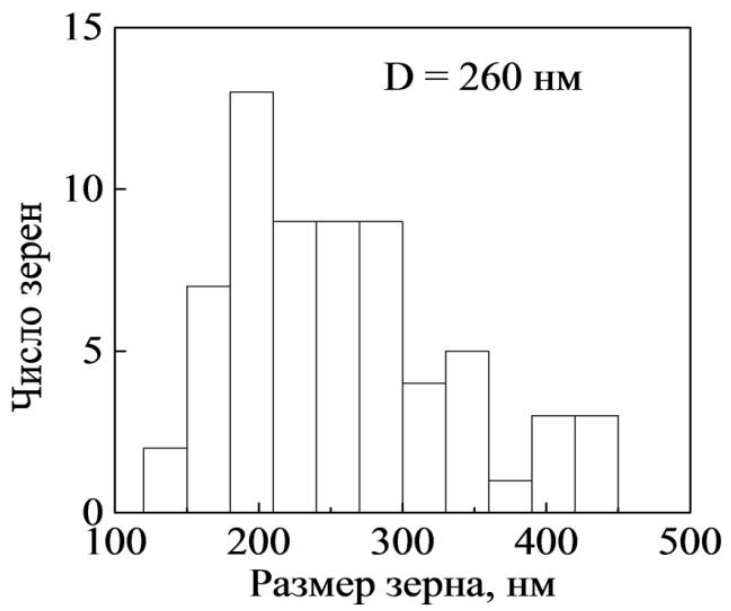

e

Рис. 9. Гистограммы распределения зерен по размерам в оловянистой бронзе после КВД на 1 и 5 оборотов и отжига при различных температурах:

$a-$ КВД на 1 оборот и отжиг при $200{ }^{\circ} \mathrm{C} ; \sigma-$ КВД на 5 оборотов и отжиг при $200{ }^{\circ} \mathrm{C}$;

в - КВД на 1 оборот и отжиг при $250{ }^{\circ} \mathrm{C} ; 2-$ КВД на 5 оборотов и отжиг при $250{ }^{\circ} \mathrm{C}$;

$\partial-$ КВД на 1 оборот и отжиг при $300{ }^{\circ} \mathrm{C} ; e-$ КВД на 5 оборотов и отжиг при $300{ }^{\circ} \mathrm{C}$ 


\section{4. Заключение}

Таким образом, влияние существенного увеличения концентрации примесных атомов в меди выражается в очевидном улучшении ее способности к наноструктурированию и к получению наноструктурного состояния уже при относительно малой степени деформации даже при одном обороте КВД при комнатной температуре. Увеличение степени деформации до 5 оборотов не дает дополнительного вклада в измельчение структуры и упрочнение, и материал выходит на стабильную стадию, когда с увеличением степени деформации размеры зерен и микротвердость не меняются уже после 1 оборота КВД.

Повышенное содержание примесей, очевидно, замедляет и релаксационные процессы. Наряду с повышенной термической стабильностью полученных наноструктур, переход от равномерного общего увеличения размеров кристаллитов при нагреве к резкому развитию рекристаллизационных процессов становится более сглаженным, чем в чистых металлах.

Влияние степени деформации на термическую стабильность получаемой структуры бронзы также не столь значительно, как в чистых металлах. Общий анализ размеров зерен указывает на несколько более высокую стабильность менее деформированного (на 1 оборот) образца, в котором релаксационные и рекристаллизационные процессы протекают несколько менее интенсивно по сравнению с образцом, продеформированным на 5 оборотов, причем наиболее заметно разница в структуре проявляется при температуре отжига $300{ }^{\circ} \mathrm{C}$.

\section{Благодарность}

Работа выполнена в рамках Государственной программы «Спин» № 01201463330 при частичной поддержке Программы фундаментальных исследований УрО РАН (проект 15-9-2-44) и РФФИ (проект 15-03-03103).

\section{Литература}

1. Valiev R. Z. Nanostructuring of metals by severe plastic deformation for advanced properties // Nature Materials. - 2004. - Vol. 3. - P. 511-516. - DOI: 10.1038/nmat1180.

2. Producing bulk ultrafine-grained materials by severe plastic deformation / R. Z. Valiev, Y. Estrin, Z. Horita, T. G. Langdon, M. J. Zehetbauer, Y. T. Zhu // The Journal of the Minerals, Metals\& Materials Society. - 2006. - Vol. 58. - Iss. 4. - P. 33-39. - DOI: 10.1007/s11837-006-0213-7.

3. Валиев Р. 3., Александров И. В. Объемные наноструктурные металлические материалы. - М. : Академкнига, 2007. - 398 с. - ISBN 978-5-94628-217-8.

4. Estrin Y., Vinogradov A. Extreme grain refinement by severe plastic deformation: A wealth of challenging science // Acta Materialia. - 2013. - Vol. 61. - Iss. 3. - P. 782-817. DOI:10.1016/j.actamat.2012.10.038.

5. Advantages and limitations of HPT: A review / R. Pippan, S. Scheriau, A. Hohenwarte, M. Hafok // Materials Science Forum. - 2008. - Vol. 584-586. - P. 16-21. DOI: $10.4028 /$ www.scientific.net/MSF.584-586.16.

6. Vorhauer A., Pippan R. Microstructure and thermal stability of tungsten based materials processed by means of Severe Plastic Deformation // Materials Science Forum. - 2003. Vol. 426-432. - P. 2747-2752. - DOI:10.4028/www.scientific.net/MSF.426-432.2747.

7. Sabirov I., Pippan R. Characterization of tungsten fragmentation in a W-25\% Cu composite after high-pressure torsion // Materials Characterization. - 2007. - Vol. 58, iss. 10. - P. 848-853. DOI: 10.1016/j.matchar.2006.08.001.

8. Structure and properties of grain boundaries in submicrocrystalline $\mathrm{W}$ obtained by severe plastic deformation / V. V. Popov, R. Z. Valiev, E. N. Popova, A. V. Sergeev, A. V. Stolbovsky, V. U. Kazihanov // Defect and Diffusion Forum. - 2009. - Vol. 283-286. - P. 629-638. DOI: 10.4028/www.scientific.net/DDF.283-286.629. 
9. Structure and properties of grain boundaries in submicrocrystalline molybdenum prepared by high-pressure torsion / V. V. Popov, G. P. Grabovetskaya, A. V. Sergeev, I. P. Mishin, A. N. Timofeev, E. V. Kovalenko // The Physics of Metals and Metallography. - 2010. - Vol. 109, iss. 5. - P. 556-562. - DOI: 10.1134/S0031918X10050169.

10. Thermal stability of nanocrystalline $\mathrm{Nb}$ produced by severe plastic deformation / E. N. Popova, V. V. Popov, E. P. Romanov, V. P. Pilyugin // The Physics of Metals and Metallography. - 2006. - Vol. 101, iss. 1. - P. 52-67. - DOI: 10.1134/S0031918X06010078.

11. Effect of the degree of deformation on the structure and thermal stability of nanocrystalline niobium produced by high-pressure torsion / E. N. Popova, V. V. Popov, E. P. Romanov, V. P. Pilyugin // The Physics of Metals and Metallography. - 2007. - Vol. 103, iss. 4. - P. 407-413. - DOI: 10.1134/S0031918X0704014X.

12. Popov V. V., Popova E. N., Stolbovskiy A. V. Nanostructuring Nb by various techniques of severe plastic deformation // Materials Science and Engineering: A. - 2012. - Vol. 539. - P. 22-29. - DOI:10.1016/j.msea.2011.12.082.

13. Schafler E., Pippan R. Effect of thermal treatment on microstructure in high pressure torsion (HPT) deformed nickel // Materials Science and Engineering: A. - 2004. - Vol. 387-389. P. 799-804. - DOI:10.1016/j.msea.2004.01.112.

14. Thermal behavior of $\mathrm{Ni}(99.967 \%$ and $99.5 \%$ purity) deformed to an ultra-high strain by high pressure torsion / H. W .Zhang, X. Huang, R. Pippan, N. Hansen // Acta Materialia. - 2010. Vol. 58, iss. 5. - P. 1698-1707. - DOI:10.1016/j.actamat.2009.11.012.

15. Evolution of the structure and hardness of nickel upon cold and low-temperature deformation under pressure / V. P. Pilyugin, T. M. Gapontseva, T. I. Chashchukhina, L. M. Voronova, L. I. Shchinova, M. V. Degtyarev // The Physics of Metals and Metallography. - 2008. - Vol. 105, iss. 4. - P. 409-419. - DOI: 10.1134/S0031918X08040157.

16. Nanostructurization of $\mathrm{Nb}$ by high-pressure torsion in liquid nitrogen and the thermal stability of the structure obtained / V. V. Popov, E. N. Popova, A. V. Stolbovskii, V. P. Pilyugin, N. K. Arkhipova // The Physics of Metals and Metallography. - 2012. - Vol. 113, iss. 3. P. 295-301. - DOI: 10.1134/S0031918X1203009X.

17. Thermal stability of nanocrystalline structure in niobium processed by high pressure torsion at cryogenic temperatures / V. V. Popov, E. N. Popova, A. V. Stolbovskii, V. P. Pilyugin // Materials Science and Engineering: A. - Vol. 528, iss. 3. - P. 1491-1496. DOI:10.1016/j.msea.2010.10.052.

18. Rathmayr G. B., Pippan R. Influence of impurities and deformation temperature on the saturation microstructure and ductility of HPT-deformed nickel // Acta Materialia. - 2011. - Vol. 59, iss. 19. - P. 7228-7240. - DOI: 10.1016/j.actamat.2011.08.023.

19. Thermal stability of nickel structure obtained by high-pressure torsion in liquid nitrogen / V. V. Popov, E. N. Popova, D. D. Kuznetsov, A. V. Stolbovskii, V. P. Pilyugin // The Physics of Metals and Metallography. - 2014. - Vol. 115, iss. 7. - P. 682-691. DOI: $10.1134 / \mathrm{S} 0031918 \mathrm{X} 14070060$.

20. Structure and thermal stability of $\mathrm{Cu}$ after severe plastic deformation / V. V. Popov, A. V. Stolbovsky, E. N. Popova, V. P. Pilyugin // Defect and Diffusion Forum. - 2010. Vol. 297-301. - P. 1312-1321. - DOI:10.4028/www.scientific.net/DDF.

21. Structure, thermal stability, and state of grain boundaries of copper subjected to highpressure torsion at cryogenic temperatures / A. V. Stolbovsky, V. V. Popov, E. N. Popova, V. P. Pilyugin // Bulletin of the Russian Academy of Sciences: Physics. - 2014. - Vol. 78, iss. 9. P. 908-916. - DOI: 10.3103/S1062873814090299.

22. Fabrication and thermal stability of a nanocrystalline $\mathrm{Ni}-\mathrm{Al}-\mathrm{Cr}$ alloy: comparison with pure $\mathrm{Cu}$ and Ni / K. Oh-ishi, Z. Horita, D. J. Smitz, R. Z. Valiev, M. Nemoto, T. G. Langdon // Journal of Materials Research. - 1999. - Vol. 14, iss. 11. - P. 4200-4207. 
23. Saturation of fragmentation during severe plastic deformation / R. Pippan, S. Scheriau, A. Taylor, M. Hafok, A. Hohenwarter, A. Bachmaier // Annual Review of Materials Research. 2010. - Vol. 40. - P. 319-343. - DOI: 10.1146/annurev-matsci-070909-104445.

24. Конькова Т. Н., Миронов С. Ю., Корзников А. В. Аномальный рост зерен в криогенно-деформированной меди // Физическая мезомеханика. - 2011. - Т. 14, № 4. - С. 29-38.

25. Konkova T. N., Mironov S. Y., Korznikov A. V. Room-temperature instability of the structure of copper deformed at a cryogenic temperature // Russian metallurgy (Metally). - 2011. Vol. 2011, iss. 7. - P. 689-698. - DOI: 10.1134/S0036029511070081.

26. Popova E. N., Romanov E. P., Sudareva S. V. A15 superconducting composites and highstrength $\mathrm{Cu}-\mathrm{Nb}$ conductors // The Physics of Metals and Metallography. - 2003. - Vol. 96, iss. 2. P. 146-159.

27. Influence of Different Deformation Techniques on the Structure of Bronze Matrix in Multifilamentary $\mathrm{Nb}_{3} \mathrm{Sn}$ Composites / E. N. Popova, L. A. Rodionova, S. V. Sudareva, E. P. Romanov, N. E. Khlebova, A. M. Chukin // The Physics of Metals and Metallography. - 1993. - Vol. 76, iss. 2. - P. 228-234.

28. Microhardness and microstructural evolution in pure nickel during high-pressure torsion / A. P. Zhilyaev, S. Lee, G. V. Nurislamova, R. Z. Valiev, T. G. Langton // Scripta Materialia. 2001. - Vol. 44, iss. 12. - P. 2753-2758. - DOI:10.1016/S1359-6462(01)00955-1.

29. Microstructure and Mechanisms of its Formation in Submicrocrystalline Copper Produced by Severe Plastic Deformation / A. N. Tyumentsev, I. A. Ditenberg, Yu. P. Pinzhin, A. D. Korotaev, and R. Z. Valiev // The Physics of Metals and Metallography. - 2003. - Vol. 96, iss. 4. P. 378-387.

30. Structure of $\mathrm{Cu}$ deformed by high pressure torsion / T. Hebesberger, H. P. Stuwe, A. Vorhauer, F. Wetscher, R. Pippan // Acta Materialia. - 2005. - Vol. 53, iss. 2. - P. 393-402. DOI: 10.1016/j.actamat.2004.09.043. 